\title{
Mechanism and prevention of acute kidney injury from cast nephropathy in a rodent model
}

\author{
Wei-Zhong Ying, ${ }^{1}$ Christopher E. Allen, ${ }^{1,2}$ Lisa M. Curtis, ${ }^{1,2}$ Kristal J. Aaron, ${ }^{1}$ and Paul W. Sanders ${ }^{1,2}$ \\ ${ }^{1}$ Division of Nephrology, Department of Medicine, Nephrology Research and Training Center, Center for Free Radical Biology, \\ and Department of Physiology and Biophysics, University of Alabama at Birmingham, Birmingham, Alabama, USA. \\ 2Department of Veterans Affairs Medical Center, Birmingham, Alabama, USA.
}

\begin{abstract}
A common renal complication of multiple myeloma is "myeloma kidney," a condition also known as cast nephropathy. The renal lesions (casts) are directly related to the production of monoclonal immunoglobulin free light chains (FLCs), which coprecipitate with Tamm-Horsfall glycoprotein (THP) in the lumen of the distal nephron, obstructing tubular fluid flow. Here, we report that analysis of the binding interaction between FLCs and THP demonstrates that the secondary structure and key amino acid residues on the complementarity-determining region 3 (CDR3) of FLCs are critically important determinants of the molecular interaction with THP. The findings permitted development of a cyclized competitor peptide that demonstrated strong inhibitory capability in the binding of FLCs to THP in vitro. When used in a rodent model of cast nephropathy, this cyclized peptide construct served as an effective inhibitor of intraluminal cast formation and prevented the functional manifestations of acute kidney injury in vivo. These experiments provide proof of concept that intraluminal cast formation is integrally involved in the pathogenesis of acute kidney injury from cast nephropathy. Further, the data support a clinically relevant approach to the management of renal failure in the setting of multiple myeloma.
\end{abstract}

\section{Introduction}

One of the functions of the kidney is to filter and metabolize low molecular weight proteins that include immunoglobulin free light chains (FLCs). Polyclonal FLCs are secreted normally in the circulation and appear in the glomerular ultrafiltrate. FLCs are reabsorbed into the proximal tubular epithelium and then hydrolyzed. In the setting of overproduction of monoclonal FLCs, a wide variety of renal pathologies can develop, including glomerular diseases, such as Amyloid Light-chain (AL-type) amyloidosis and monoclonal light chain deposition disease, or tubular damage, known as proximal tubulopathy (1-5). In addition, FLCs that escape tubular reabsorption are presented to the distal nephron and, in the proper conditions, form intraluminal casts that obstruct tubular fluid flow (3, 6-8). Clinical manifestations of this phenomenon, known as cast nephropathy, include acute kidney injury (AKI) and progressive renal failure. Because this complication occurs in multiple myeloma, which constitutes $12 \%-13 \%$ of hematologic malignancies in the US (9), the term "myeloma kidney" has also been used. Cast nephropathy is a seminally important and common complication in myeloma, since reduced renal function contributes to morbidity and mortality and limits therapeutic options (10-12). At the time of presentation, nearly half of these patients have renal dysfunction, as defined by a serum creatinine concentration greater than or equal to $1.3 \mathrm{mg} / \mathrm{dl}(10)$. When kidney tissue was examined histologically, cast nephropathy was the major cause of renal failure (13).

Prior studies determined an important role for Tamm-Horsfall glycoprotein (THP) in cast nephropathy (7). THP possesses a single FLC-binding domain, termed LCBD $(14,15)$, and the comple-

Conflict of interest: The authors have declared that no conflict of interest exists. Citation for this article: J Clin Invest. 2012;122(5):1777-1785. doi:10.1172/JCI46490. mentarity-determining region 3 (CDR3) of most FLCs tested specifically interacted with this site (16). The following experiments were designed to analyze the binding interaction between FLCs and THP and to test the hypothesis that a competitive inhibitor of the interaction between THP and monoclonal FLCs prevents AKI induced in cast nephropathy.

\section{Results}

The CDR3 of FLCs demonstrated varying binding affinities to THP. Previous publications demonstrated that FLCs bind to a specific domain on human THP, but possess variable affinities for THP $(14,15)$. Initial experiments expanded the original studies by using the variable light chain $\left(V_{L}\right)$ domain of 20 unique human FLCs from the $\lambda \mathrm{I}, \lambda \mathrm{III}, \lambda \mathrm{IV}, \lambda \mathrm{V}, \lambda \mathrm{VI}, \kappa \mathrm{I}, \kappa \mathrm{II}$, and $\kappa \mathrm{IV}$ families. The yeast 2-hybrid system originally designed by Fields and Song (17) was employed to determine the site on the light chain that interacted with THP (16). The binding interactions of these $\kappa$ and $\lambda$ FLCs with recombinant 26-residue and 263-residue fragments of THP, which contained the previously described LCBD, were quantified. The findings were similar when either the smaller or larger THP fragment was used, so the data presented in this paper are from experiments that used the larger fragment (Table 1). All tested families of FLCs bound to THP, with members of the $\lambda \mathrm{V}$ family demonstrating the lowest binding affinity. The relative strength of the interactions differed among the 20 different FLCs (Table 1). The variable domain of the $\lambda$ V FLCs, LKPBLL53, showed the lowest affinity interaction: yeast transformed with this construct did not grow in leucine-deficient medium and possessed low $\beta$-gal activity. The intact $V_{L}$ of the $\lambda$ IIIa FLCs, ITPBLL86, demonstrated the highest binding affinity among the FLCs tested. A series of truncation mutations performed on the FLCs again confirmed that the CDR 3 of both $\kappa$ and $\lambda$ FLCs specifically interacted with the 


\section{Table 1}

Binding affinities of 20 different FLCs with THP

\begin{tabular}{|c|c|c|}
\hline $\begin{array}{l}\text { Deduced } \\
\text { Insert }\end{array}$ & $\begin{array}{l}\text { Relative } \\
3 \text { amino acid sequence }\end{array}$ & reactivity \\
\hline \multicolumn{3}{|l|}{$\begin{array}{l}\lambda \text { FLCs } \\
\lambda \mid \text { subgroup }\end{array}$} \\
\hline LBPBLL2N & QSYDNTLSGSYV & $6.7 \pm 1.0$ \\
\hline \multicolumn{3}{|l|}{$\lambda$ III subgroup } \\
\hline ITPBLL56 (גIII) & QVWDNSVGV & $7.1 \pm 1.3$ \\
\hline ITPBLL2 ( $\lambda$ III) & QVWHSSSDHYV & $2.0 \pm 0.2$ \\
\hline LBPBLL2 ( $\lambda$ III) & QSADNSGTFWI & $6.8 \pm 0.8$ \\
\hline ITPBLL79 (גIII) & QSADSSGTYWV & $6.0 \pm 2.1$ \\
\hline 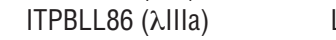 & LSADSSGSYLYV & $7.8 \pm 0.9$ \\
\hline LKPBLL68 ( $\lambda$ III) & YSATDNNWV & $6.3 \pm 0.6$ \\
\hline ITPBLL11 ( $\lambda$ IIIb) & QSTDSSGTYR & $3.4 \pm 0.3$ \\
\hline ITPBLL22 ( $\lambda$ IIIc) & QAWDRSTVV & $4.9 \pm 0.7$ \\
\hline \multicolumn{3}{|l|}{$\lambda$ IV subgroup } \\
\hline LBPBLL2Q $(\lambda \mathrm{IV})$ & ETWDSDTRV & $6.5 \pm 0.8$ \\
\hline 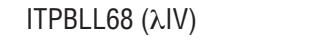 & QTWDTGFWV & $5.6 \pm 1.0$ \\
\hline \multicolumn{3}{|l|}{$\lambda V$ subgroup } \\
\hline ITPBLL69 $(\lambda V)$ & AMWYSDVYV & $2.0 \pm 0.4^{\mathrm{A}}$ \\
\hline LKPBLL53 $(\lambda V)$ & MIRGI & $1.6 \pm 0.4^{\mathrm{A}}$ \\
\hline LBPBLL7 (גV) & LIWHSRAYV & $2.9 \pm 0.4$ \\
\hline \multicolumn{3}{|l|}{$\lambda V I$ subgroup } \\
\hline ITPBLL75 ( $\lambda \mathrm{VI})$ & VSLMMAGIIMS & $4.8 \pm 0.6$ \\
\hline LBPBLL2S $(\lambda V I)$ & QSSDTTNQV & $5.8 \pm 0.8$ \\
\hline \multicolumn{3}{|l|}{$\kappa \mathrm{FLCs}$} \\
\hline \multicolumn{3}{|l|}{ кl subgroup } \\
\hline \multicolumn{3}{|l|}{ кll subgroup } \\
\hline SSH23 (kll) & MQGTHWPPLT & $4.9 \pm 0.7$ \\
\hline ITPBL11 (кII) & QQYGSSPCT & $3.4 \pm 0.7$ \\
\hline \multicolumn{3}{|l|}{ кIV subgroup } \\
\hline BCSyn9 (кIV) & QQLNSYPFT & $3.2 \pm 1.0$ \\
\hline $\begin{array}{l}\text { Positive control } \\
\text { (pGBKT7-53/pGADT7-T) }\end{array}$ & - & $12.7 \pm 2.1$ \\
\hline $\begin{array}{l}\text { Negative control } \\
\quad(\text { pGBKT7-Lam/pGADT7-T) }\end{array}$ & - & $1.0 \pm 0.2^{\mathrm{A}}$ \\
\hline $\begin{array}{l}\text { pGBKT7-THP with pGADT7 } \\
\text { (empty vector) }\end{array}$ & - & $0.9 \pm 0.2^{A}$ \\
\hline
\end{tabular}

ADid not interact under stringent conditions. $n=3-9$ experiments in each group.

THP constructs. Reactivity with THP correlated weakly $\left(R^{2}=0.23\right.$; $P=0.02)$ with the number of amino acid residues in the CDR3.

Key amino acid residues in the CDR3 of FLCs determined binding to THP. An artificial construct was designed using framework 2 and framework 3 of LKPBLL53, an FLC that did not interact significantly with THP (Table 1). Various CDR3 sequences were then inserted into this construct (Figure 1). The construct that did not contain an insert did not interact with THP in the yeast 2-hybrid assay. The construct that contained the CDR3 of ITPBLL86 (LSADSSGSYLYV) showed the greatest interaction, followed by the CDR3 (QVWDSTSDHYV) of ITPBLL1. The CDR3 of LKPBLL53 interacted least well with THP (Figure 1B). The CDR3 of ITPBLL2, which consisted of QVW $\underline{H} S \underline{S} S D H Y V$, differed from ITPBLL1 by only 2 amino acid residues (underlined), but demonstrated markedly reduced binding affinity in this assay. The importance of these residues in binding was further determined by mutating residues in the ITPBLL86 CDR3 peptide construct (Figure 2). Changing the aspartate to a hydrophobic amino acid residue greatly inhibited binding, which was somewhat reduced further by changing 2 of the serine residues to phenylalanine.

A cyclized, synthetic peptide inbibited binding between FLCs and THP in vitro. Using the data from Table 1, we synthesized 2 peptides (AHX-LSADSSGSYLYVF and QSYDNTLSGSYVF) based upon CDR3 peptide sequences known to interact strongly with THP. These peptides were then used to determine whether they prevented binding between FLCs and THP in a competitive ELISA (16). Both peptides dose dependently inhibited FLCs binding to THP, with a mean IC $_{50}$ of peptide 1 (AHX-LSADSSGSYLYVF) of $38.3 \pm 5.8 \mathrm{nM}$ and mean $\mathrm{IC}_{50}$ of peptide 2 (QSYDNTLSGSYVF) of $55.0 \pm 16.1 \mathrm{nM}$ (Figure 3 ). In an effort to improve the inhibitory capability, both synthetic peptides were cyclized. Cysteine residues were added to the termini of both peptides, then cyclized through intramolecular disulfide bonding between these cysteine residues. When comparing the $\mathrm{IC}_{50}$ to that of the linear peptides, cyclized peptide $1\left(\mathrm{IC}_{50}=10.0 \pm 2.6 \mathrm{nM}\right)$ and cyclized peptide $2\left(\mathrm{IC}_{50}=6.7 \pm 2.3 \mathrm{nM}\right)$ demonstrated a markedly increased $(P<0.05)$ inhibitory capability in the same assay.

In additional studies, the inhibitory potential of synthetic cyclized peptide 1 (AHX-CLSADSSGSYLYVCKK) was compared with that of the same peptide with 2 phenylalanine substitutions (AHX-CLSAFSFGSYLYVCKK). These substitutions led to significantly reduced $(P<0.05)$ binding to THP (Figure 2$)$. As opposed to the phenylalanine mutant form, the cyclized CDR3-mimetic peptide (AHX-CLSADSSGSYLYVCKK) served as a highly effective inhibitor that prevented, in a dose-dependent fashion, the binding of 6 different human FLCs to human THP (Figure 4).

Overlay assays were performed using human THP purified from urine and a monoclonal FLC or $\mathrm{V}_{\mathrm{L}}$ domain that had been enzymatically cleaved from the same FLCs and purified (Figure 5A). THP bound to monomeric and dimeric forms of an intact human FLC and the $\mathrm{V}_{\mathrm{L}}$ domain. To generate the biological reagents used in experiments illustrated in the bottom panel, a cell-free system was employed to generate THP and the $V_{L}$ domain of ITPBLL86. Cyclized peptide 1 (AHX-CLSADSSGSYLYVCKK) dose dependently inhibited binding of the biotin-labeled $V_{L}$ domain of ITPBLL86 to recombinant full-length THP (Figure 5B). This same cyclized peptide 1 promoted a dose-dependent inhibition of binding of biotinylated THP to 22 FLC present on the membrane. In contrast, a synthetic cyclized control peptide (AHXCLSAHSSGSYLYVCKK), which was identical to cyclized peptide 1 except for a substitution of histidine for the aspartate at the fifth residue, did not inhibit binding of biotinylated THP to the FLCs at the concentrations tested (Figure 5C).

The cyclized competitor peptide 1 (AHX-CLSADSSGSYLYVCKK) prevented AKI from cast nephropathy. The synthetic cyclized competitor peptide 1 (AHX-CLSADSSGSYLYVCKK) was tested in vivo in a rodent model of AKI from cast nephropathy, which employed intraperitoneal administration of $20 \mathrm{mg}$ of cast-forming human $\kappa 3$ and $\lambda 2$ FLCs. Either the competitor peptide ( $8 \mathrm{mg})$, or vehicle alone was injected intraperitoneally 2 hours prior to the initial injection of the FLCs and again 24 hours following the FLC injection. Mean baseline serum creatinine concentration was $0.32 \pm 0.02$ $\mathrm{mg} / \mathrm{dl}$ in this series of experiments. While the $\lambda 2 \mathrm{FLC}$ was from a patient with biopsy-proven cast nephropathy and produced AKI with associated cast formation, the $\kappa 3$ FLC was particularly nephrotoxic and produced severe renal failure with very low urine output and weight loss over the 48 hours of follow-up (Table 2). When examined histologically, kidney tissue from vehicle-treated animals 


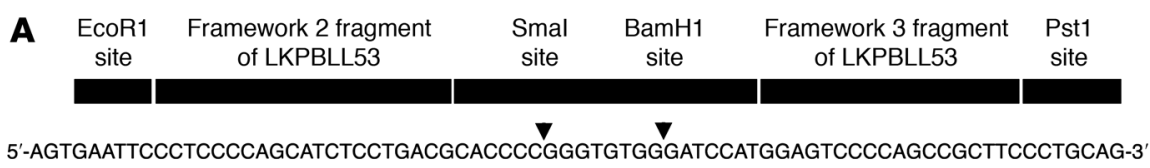

Fragment removed during insertion of CDR3 of interest

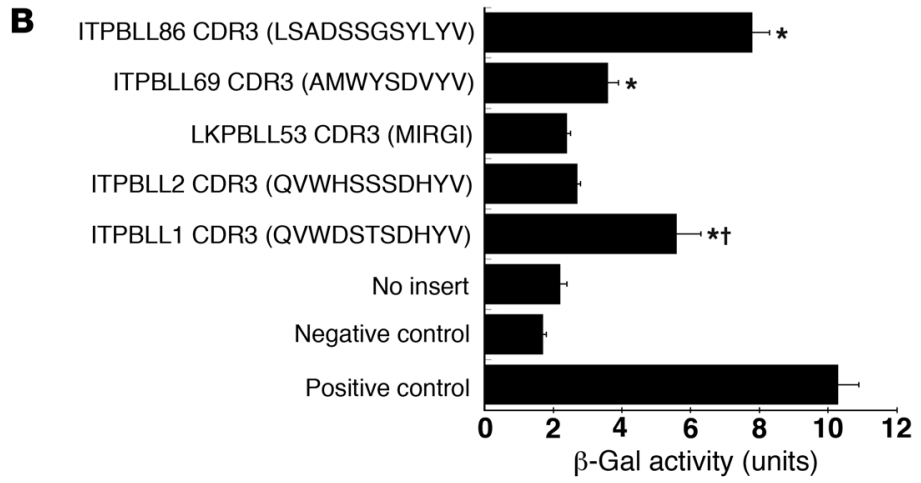

Figure 1

Key amino acid residues in the CDR3 of the $V_{L}$ domain determine binding to THP. A construct that permitted ligation of the CDR3 of interest between portions of framework 2 and framework 3 from an FLC (LKPBLL53) that did not interact with THP was used to test the interaction of the CDR3 with THP (A). As predicted, the construct that did not contain a CDR3 insert (no insert) did not interact with THP in the yeast 2-hybrid assay (B). Also as expected, the CDR3 from LKPBLL53 did not interact with THP. Binding was strongest with CDR3 (LSADSSGSYLYV) from ITPBLL86. Binding was also demonstrated with the use of the CDR3 (QVWDSTSDHYV) from ITPBLL1, but the interaction was markedly reduced with the use of the CDR3 (QVWHSSSDHYV) from ITPBLL2. These regions differ in only 2 amino residues, which are underlined in the figure. ${ }^{*} P<0.05$, compared with no insert; ${ }^{\dagger} P<0.05$, compared with ITPBLL2 CDR3 construct. $n=6-8$ experiments in each group. Data are shown as mean \pm SEM.

showed intraluminal cast formation (Figure 6). Coadministration of the competitor peptide, but not the vehicle alone, with either monoclonal FLCs completely prevented the functional manifestations of AKI. In these studies, there was a statistically significant difference between casts visualized in histological samples for animals injected with FLCs plus vehicle when compared with those injected with FLCs plus peptide. Experiments that used the $\kappa 3$ FLC yielded a Kruskal-Wallis $\chi^{2}$ test of $43.35(P<0.0001)$ and, for experiments that used the $\lambda 2$ FLC, the Kruskal-Wallis $\chi^{2}$ test was $5.16(P=0.02)$. In the group that received the $\kappa 3$ FLC, the mean

Figure 2

Mutational analysis of the CDR3 sequence demonstrated the importance of the primary sequence, in particular the fourth amino acid residue, of the CDR3 in binding to THP. ${ }^{*} P<0.05$, compared with no insert. $n=7$ experiments in each group. Data are shown as mean \pm SEM. numbers of casts in the vehicle-treated rats were $33.6 \pm 1.5$ and $11.5 \pm 1.0$ in peptidetreated rats; the median cast count was 33 (interquartile range, 28-37) for vehicle and 10 (interquartile range, 7-15) for peptide. In the group that received the $\lambda 2$ FLC, the mean numbers of casts present in the medulla were $2.5 \pm 1.2$ for vehicle-treated rats and $0.3 \pm 0.09$ for peptide-treated rats; the median cast count was 0.5 (interquartile range, $0-2$ ) for vehicle and 0 (interquartile range, $0-1$ ) for peptide.

In a rescue experiment, rats were initially injected intraperitoneally with a monoclonal FLC (к2), $0.25 \mathrm{mg} / \mathrm{g}$ BW. Four hours later, rats received an intraperitoneal injection of vehicle alone ( $n=6$ rats), experimental peptide 1 (AHX-CLSADSSGSYLYVCKK) ( $n=5$ rats), or control peptide (AHX-CLSAHSSGSYLYVCKK) ( $n=5$ rats), which did not inhibit binding between the к2 FLC and THP (Figure 5C). The dose of peptide was $0.1 \mathrm{mg} / \mathrm{g} \mathrm{BW}$. On the second day of the study, the animals received a second intraperitoneal injection of the experimental or control peptide, $0.1 \mathrm{mg} / \mathrm{g} \mathrm{BW}$, or vehicle alone. At the initiation of the study, BWs among the 3 groups did not differ (data not shown). By the end of the study, however, mean BW (102 $\pm 3 \mathrm{~g})$ of the group that received experimental peptide 1 (AHX-CLSADSSGSYLYVCKK) exceeded $(P<0.05)$ mean BW of the vehicle-treated $(81 \pm 12 \mathrm{~g})$ and control peptide-treated (AHX-CLSAHSSGSYLYVCKK) (84 $\pm 3 \mathrm{~g}$ ) rats. One animal in the vehicle-treated group died on the afternoon of the day after the injection of the FLCs. Compared with the group treated with experimental peptide 1 , mean serum creatinine levels increased $(P<0.05)$ in the vehicle-treated and control peptide-treated rats. When compared with mean baseline serum creatinine levels, the slight increase in serum creatinine of the experimental peptide 1 -treated rats did not reach statistical significance (Figure 7). Quantitative analysis of casts visualized in histological samples in this series of studies demonstrated a statistically significant difference between the treatment groups (Kus-

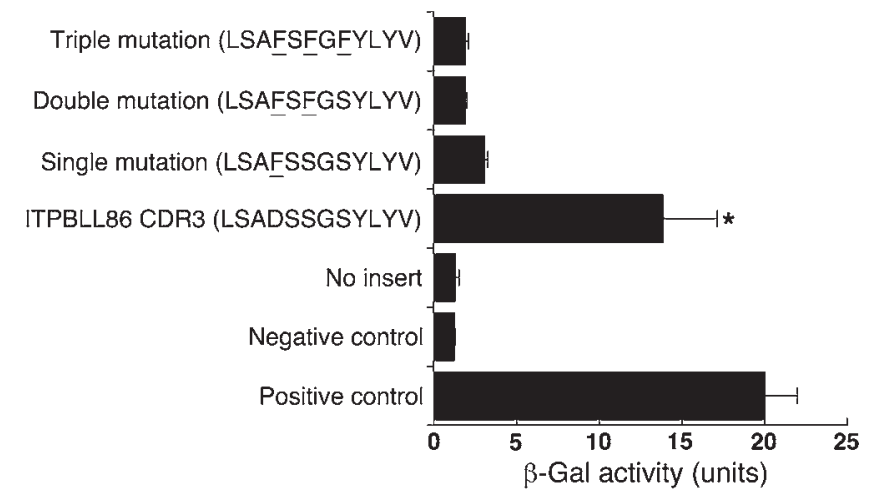



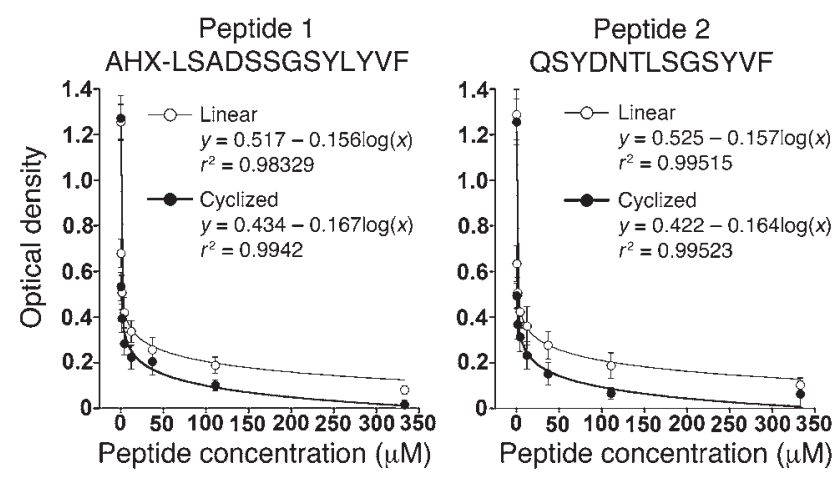

kal-Wallis $\chi^{2}$ test of $\left.81.0 ; P<0.0001\right)$. Among the 3 treatment types, the mean number of casts was $6.2 \pm 0.64$ for vehicle, $2.8 \pm 0.46$ for the control peptide, and $0.38 \pm 0.16$ for the experimental peptide; the median cast count by injection type was 5 (interquartile range, 4-7) for vehicle, 2 (interquartile range, 0-4) for control peptide, and 0 (interquartile range, $0-0$ ) for the experimental peptide.

\section{Discussion}

In the present studies, multiple strategies were used to demonstrate that (a) the secondary structure and key amino acid residues on the CDR3 of FLCs were important determinants of the molecular interaction with THP; and (b) a cyclized peptide construct that binds strongly to THP served as an effective inhibitor of cast nephropathy in vivo. The present biochemical and in vivo experiments therefore offered a proof of concept that intraluminal cast formation was integrally involved in the pathogenesis of AKI from cast nephropathy. Further, the data suggested a clinically important and, we believe, novel approach to the management of AKI in this setting.

THP consists of 5 known domains that include an EGF-like domain and 2 calcium-binding EGF-like domains at the $\mathrm{N}$ terminus, 2 zona pellucida domains (ZP-N and $\mathrm{ZP}-\mathrm{C}$ ) at the $\mathrm{C}$ terminus, and a central region, recently termed $\mathrm{D} 8 \mathrm{C}$, that is also present in the pancreatic glycoprotein GP2 and liver-specific ZP domain-con-

\section{Figure 3}

Graphs of the effect of competitor peptides and cyclized competitor peptides on binding of THP to FLCs. While both CDR3 mimetic peptides inhibited binding of FLCs to THP, cyclization, which was accomplished through intramolecular disulfide bonding between cysteine residues added onto the amino and carboxyl termini, shifted the inhibition curves to the left. $n=5$ experiments at each concentration in each group. Data are shown as mean \pm SEM. taining protein (Figure 8 and refs. 18-22). In addition, 4 potential isoforms of THP arise from alternative splicing $(23,24)$. These include the canonical or full-length protein (isoform no. 1); isoform no. 2, which lacks both calcium-binding EGF-like domains and the first 50 residues of the central region; and isoform no. 3 , which has a 30-residue deletion shortly thereafter. This 30-residue stretch is replaced with a single proline, most likely leading to a sharp bend, and maintaining the overall conformation of the molecule. A fourth isoform that contains a 22-residue deletion within the second calcium-binding EGF-like domain has also been suggested. Prior studies identified a single FLC-binding domain (LCBD) on THP where FLCs bind $(14,15)$. The LCBD of THP was within the $\mathrm{D} 8 \mathrm{C}$ region just downstream of the last residue absent from isoform no. 3 (Figure 8). The alternative splicing data further inform the intramolecular disulfide bonding state and connectivity patterns, which were predicted using 2 peer-reviewed online neural networks (DISULFIND and DiANNA) $(25,26)$. A total of 19 cysteines were found in the unknown central region. Taking advantage of the alternative splicing patterns, the cysteines were reduced to 3 groups. Eighteen cysteines were found in the deleted region of isoform no. 2 . Twelve of them were located within the 2 calcium-binding EGF-like domains with known cysteine-binding patterns. The remaining 6 cysteines deleted in isoform no. 2 were considered to form 1 group, as were the 2 cysteines deleted

\section{Figure 4}

The cyclized peptide (AHX-CLSADSSGSYLYVCKK) (circles) was a highly effective inhibitor that completely prevented the binding of THP to 6 different human monoclonal FLCs. The control peptide (AHXCLSAFSFGSYLYVCKK) (white squares) did not effectively inhibit binding of THP to any of the 6 FLCs. $n=4$ experiments in each group. Data are shown as mean \pm SEM.
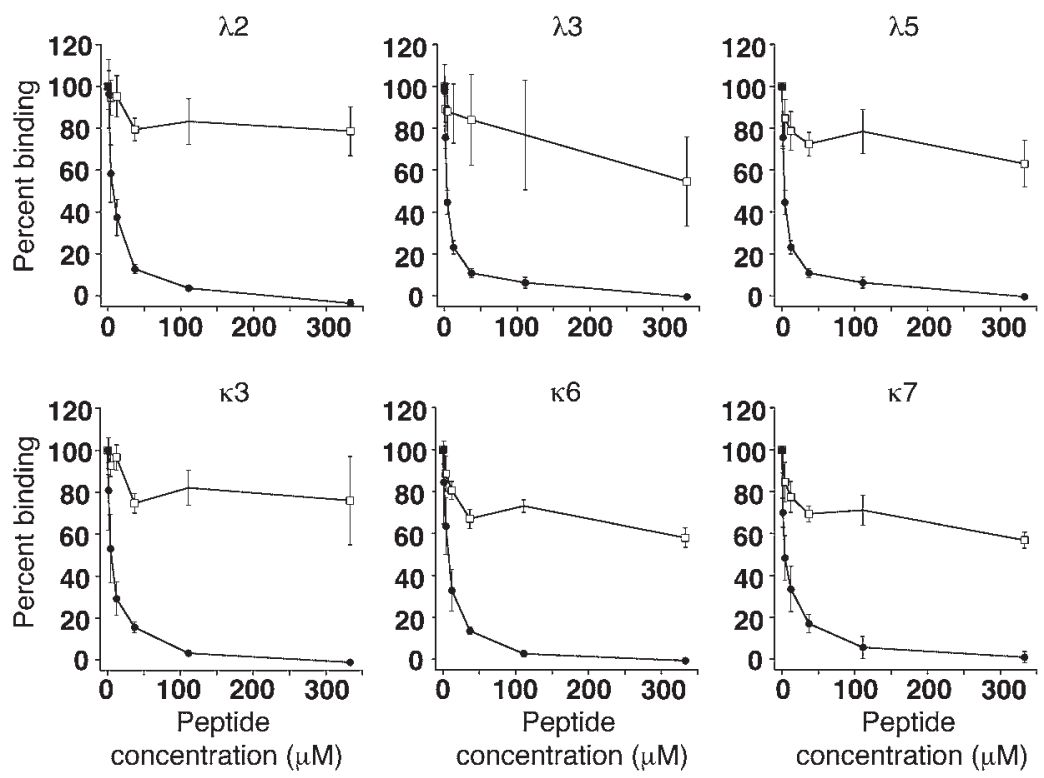

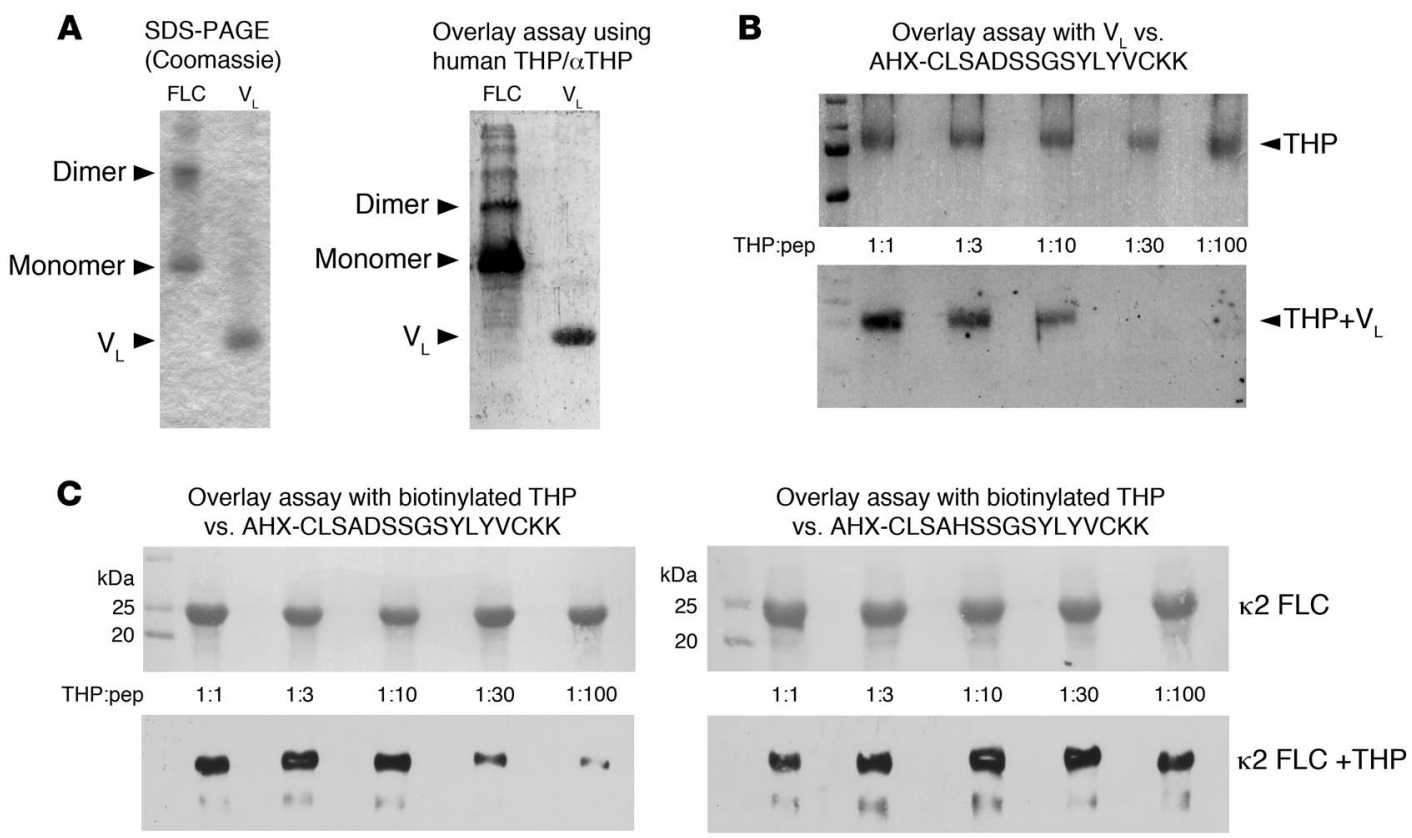

\section{Figure 5}

Overlay (far-Western) assays demonstrating the interaction between FLCs and THP. (A) Coomassie-stained gel (left) depicts the monomeric and dimeric forms of a monoclonal FLC and the $V_{L}$ domain, which had been enzymatically cleaved from the FLCs. The adjacent blot (right) demonstrates that human THP bound to monomers, dimers, and polymeric FLCs, as well as the $V_{L}$ domain of the FLCs. (B) Results of an experiment that used cyclic peptide 1 (AHX-CLSADSSGSYLYVCKK) to compete with the biotinylated $V_{L}$ domain of FLC ITPBLL86 for binding to THP immobilized onto PVDF membrane. The reversible protein stain demonstrated the presence of THP in each lane (top). Addition of increasing molar amounts, relative to THP, of the cyclic peptide prevented binding of the $V_{L}$ domain to THP. (C) Results of an overlay assay that used cyclized peptide 1 (AHX-CLSADSSGSYLYVCKK) or a cyclized control peptide (AHX-CLSAHSSGSYLYVCKK) to compete with biotinylated THP for binding to $\kappa 2$ FLC immobilized onto PVDF membrane. The reversible protein stain demonstrated the presence of $\kappa 2$ FLC in each lane (top). Cyclized peptide 1, but not the cyclized control peptide, dose dependently inhibited binding of THP to the к2 FLC (bottom).

in isoform no. 3. This grouping left 11 of the original 19 cysteines remaining within the unknown central region. Interestingly, the first 2 cysteines of this group were considered paired by all prediction patterns tested. They were the first and eighth residues of the LCBD of THP (ㄷHWSGHC), and their binding led to the formation of a tight loop. A molecular model of the cyclic peptide (CAHWSGHC) was generated using GAMESS and TINKER (27-29). The most common rotamers were selected, except when orbital overlap prevented it. Charge and multiplicity were assigned, and the data were sent to GAMESS for energy minimization initially in vacuo and then solvated in a water box. Energy minimization was then carried out in TINKER using Amber94 as the parameter file. The model depicts the terminal cysteines paired to form a loop structure that forces the 2 histidine residues into close proximity. The model further predicts the potential for strong ionic interaction between the histidines on THP and negatively charged residues on the FLCs, a finding demonstrated in the present series of studies. The data fit well with prior studies showing the effect of $\mathrm{pH}$ on binding of FLCs to THP (30) and prevention of AKI from monoclonal FLCs (31). This model also correlates well with the yeast 2-hybrid data in the present study and provides new insights into the structure of the LCBD. As predicted from this model and the data presented, the charge of the THP-binding peptide, particularly at the fourth residue, was a critical determinant of binding, since replacing the aspartate with histidine inhibited the binding interaction.

In designing a competitor that inhibited binding between THP and any FLCs, a major consideration became the site of interaction in vivo. While a competitor that mimicked the LCBD of

\section{Table 2}

Effect of parenteral administration of competing cyclic peptide versus vehicle on renal function following exposure to 2 nephrotoxic FLCs

\begin{tabular}{|c|c|c|c|c|c|c|}
\hline Group & $\begin{array}{l}\text { Initial } \\
\text { BW (g) }\end{array}$ & $\begin{array}{l}\text { Final } \\
\text { BW }(g)\end{array}$ & $\begin{array}{l}\text { BW change } \\
\text { (g) }\end{array}$ & $\begin{array}{c}\text { Final BUN } \\
(\mathrm{mg} / \mathrm{dl})\end{array}$ & $\begin{array}{c}\text { Final serum } \\
\text { creatinine }(\mathrm{mg} / \mathrm{dl})\end{array}$ & $\begin{array}{c}\text { Urine flow rate } \\
(\mu \mathrm{l} / \mathrm{min} \text { per } 100 \mathrm{~g} \mathrm{BW})\end{array}$ \\
\hline \multicolumn{7}{|l|}{$\kappa 3$ FLC } \\
\hline$+\mathrm{CP}$ & $79 \pm 2$ & $86 \pm 3$ & $7 \pm 2$ & $15 \pm 5$ & $0.39 \pm 0.03$ & $1.9 \pm 0.1$ \\
\hline +Vehicle & $74 \pm 2$ & $67 \pm 3$ & $-7 \pm 2$ & $47 \pm 7$ & $2.38 \pm 0.16$ & $0.09 \pm 0.04$ \\
\hline$P$ value & 0.231 & 0.008 & 0.01 & 0.02 & 0.0003 & $<0.0001$ \\
\hline \multicolumn{7}{|l|}{$\lambda 2 \mathrm{FLC}$} \\
\hline$+\mathrm{CP}$ & $130 \pm 3$ & $150 \pm 4$ & $18 \pm 4$ & $13 \pm 2$ & $0.46 \pm 0.02$ & $1.5 \pm 0.4$ \\
\hline +Vehicle & $130 \pm 1$ & $146 \pm 2$ & $8 \pm 2$ & $52 \pm 5$ & $1.30 \pm 0.07$ & $2.3 \pm 0.7$ \\
\hline$P$ value & 0.811 & 0.361 & 0.098 & 0.003 & 0.0003 & 0.355 \\
\hline
\end{tabular}

$\mathrm{CP}$, cyclic peptide. 

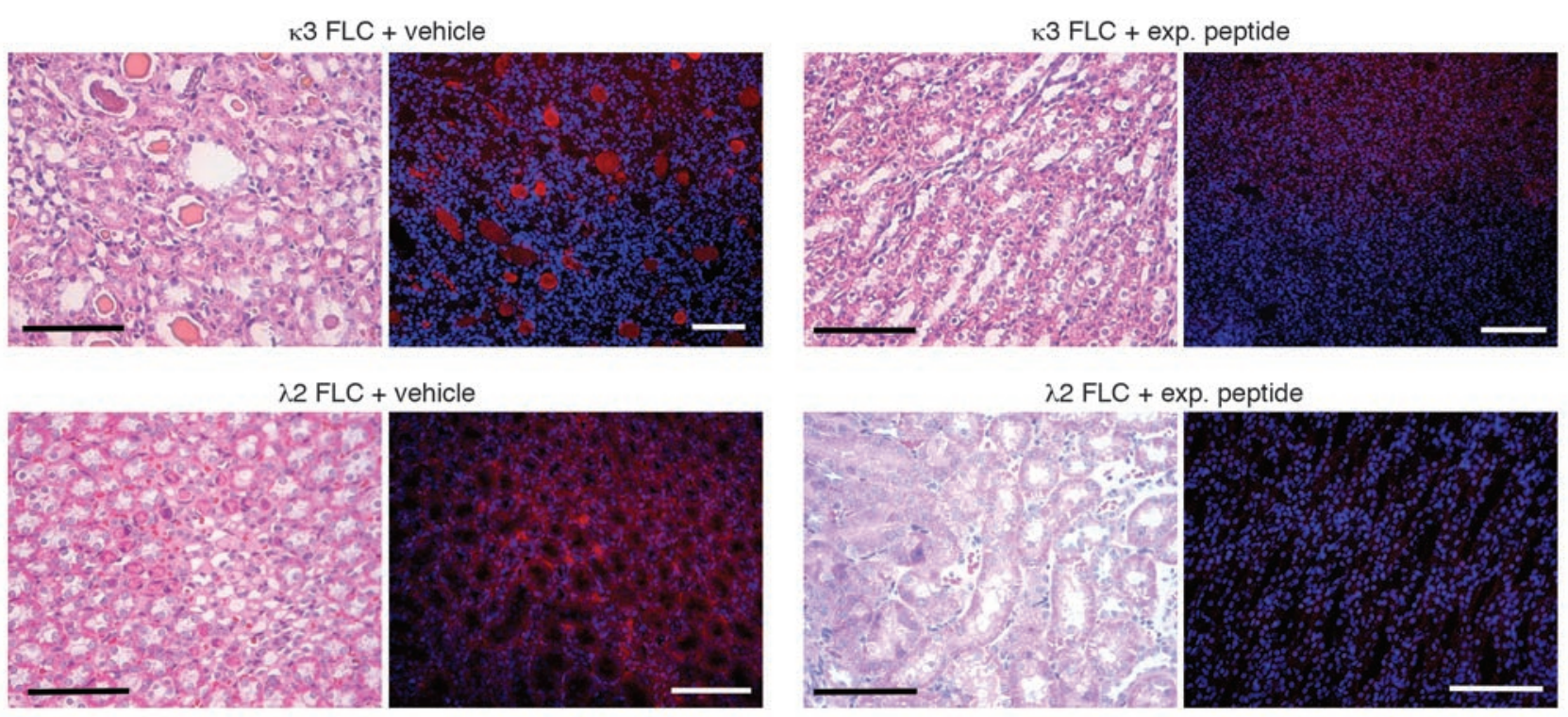

\section{Figure 6}

Light and immunofluorescence micrographs depicting the beneficial effect of the synthetic, cyclized competitor peptide in preventing cast nephropathy in vivo. Rats were injected intraperitoneally with the cyclic peptide (AHX-CLSADSSGSYLYVCKK), 8 mg, or vehicle 2 hours prior to injection with 1 of 2 unique monoclonal FLCs. Both were obtained from patients who had myeloma and renal dysfunction compatible with cast nephropathy. The patient who donated the $\lambda 2 \mathrm{FLC}$ had biopsy-proven cast nephropathy. Addition of the cyclic peptide prevented cast formation and accumulation of the monoclonal FLCs in the kidney, as demonstrated by H\&E staining and by immunofluorescent labeling of human $\kappa$ or $\lambda$ light chains, respectively, using specific primary antibodies with Texas red-labeled secondary antibody. Nuclei were visualized in the fluorescent images by DAPI staining. $n=3$ rats in each group. Scale bars: $100 \mu \mathrm{m}$.

THP might work in vivo, the initial site of interaction would be in the circulation, since an early LCBD mimetic bound to the FLCs (15). In addition, this competitor would also likely bind to light chain components of immunoglobulins. For this reason, a careful analysis of the CDR3 was undertaken in the present study and permitted the development of a competitor peptide that targeted THP. Cyclization of the peptide likely permitted the formation of a loop structure that further increased the inhibitory capability of the peptide. This cyclized competitor peptide proved to be highly efficacious in vivo (Table 2 and Figure 6). In addition, this peptide prevented AKI even when administered hours after the nephrotoxic monoclonal FLCs (Figure 7).

The level of renal function is a critical determinant of prognosis in multiple myeloma $(12,32)$, and cast nephropathy from monoclonal FLCs is the most common cause of renal dysfunction in this setting (13). When renal failure is defined as serum creatinine greater than or equal to $1.3 \mathrm{mg} / \mathrm{dl}$, nearly half of these patients have this complication (10). Approximately $22 \%$ have moderate to severe renal failure (i.e., serum creatinine above $2 \mathrm{mg} / \mathrm{dl}$ ) at presentation, with about $8 \%$ of patients requiring renal replacement therapy (12). With successful treatment, renal function normalized in $31 \%$ to $58 \%$ of patients $(32,33)$; recovery of renal function correlated with the degree of FLC proteinuria (32). Casts can also disappear with successful removal of the monoclonal FLCs (34). Along with data showing that recovery of renal function portends improved outcomes (12), these observations have led investigators to promote aggressive therapies to remove circulating FLCs, with significant improvement in renal function documented using these techniques despite severe renal injury at the initiation of treatment $(13,35)$. The present study further demonstrates the therapeutic potential of targeting the underlying pathogenetic mechanism of cast nephropathy. By providing an inhibitor of cast formation, there is opportunity to protect functioning nephrons from new cast formation and prevent AKI as well as progressive renal failure, while chemotherapy or other measures are applied to lower systemic FLC concentrations at levels that are safe for the kidney. Because cast nephropathy is the most common form of renal injury and is potentially reversible, this approach alone may offer substantial therapeutic benefit for patients who have multiple myeloma and overproduce monoclonal FLCs.

\section{Methods}

Reagents. QSYDNTLSGSYVF, CQSYDNTLSGSYVFC, AHX-LSADSSGSYLYVF, AHX-CLSADSSGSYLYVCKK, AHX-CLSAFSSGSYLYVCKK, and AHX-CLSAHSSGSYLYVCKK were synthesized commercially (Open Biosystems Products, Thermo Fisher Scientific; Biomatik Corp.). 6-Aminohexanoic acid (AHX) was added to the amino terminus of the last 4 peptides, and 2 lysine residues were added to the carboxyl terminus of the last 3 peptides to increase solubility. Peptides were cyclized by adding cysteine residues near the amino and carboxyl termini of the peptide and forming a disulfide bridge. Purity was confirmed using both mass spectrometry and HPLC.

Human THP and FLCs were purified from urine in standard fashion (6, $7,14-16,30)$. Purified human THP was biotinylated as described previously (36), using sulfo-N-hydroxysuccinimide biotin (ImmunoPure SulfoNHS-Biotin; Pierce Protein Products, Thermo Fisher Scientific), followed by dialysis against PBS at $4^{\circ} \mathrm{C}$ for 24 hours to remove free biotin. Seven different FLCs $(4 \kappa$ and $3 \lambda$ arbitrarily named $\kappa 2, \kappa 3, \kappa 6, \kappa 7, \lambda 2, \lambda 3$, and $\lambda 5$ ) were used in this study and were obtained from the urine of patients who had FLC proteinuria and clinical renal failure. Patients who donated $\lambda 2$ and $\lambda 5$ had biopsy-proven cast nephropathy. Patients who donated $\lambda 3, \kappa 2, \kappa 3$, and $\kappa 7$ had clinical presentations that were compatible with 
A

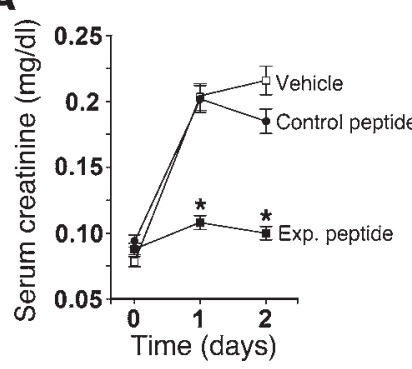

B

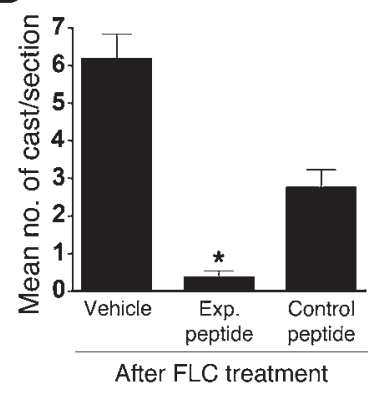

Figure 7

Experimental peptide 1 served as an effective inhibitor of cast nephropathy in vivo. Changes in (A) mean serum creatinine, determined by tandem mass spectrometry, and (B) medullary cast formation of 3 groups of rats ( $n=5-6$ per group) were determined in a rescue experiment in which the competitor cyclized experimental (exp.) peptide 1 (AHX-CLSADSSGSYLYVCKK), a control cyclized peptide (AHX-CLSAHSSGSYLYVCKK), or vehicle alone was administered intraperitoneally 4 hours after rats received the nephrotoxic FLC (к2). Mean serum creatinine increased in the vehicle-treated and control cyclized peptide-treated groups, but rats given competitor cyclized peptide 1 were protected from AKI. Animals that received experimental peptide 1 had fewer casts in the medulla compared with animals that received either vehicle or the control peptide. Rats that received the control peptide also had fewer casts $(P<0.05)$ than animals that received vehicle alone. ${ }^{*} P<0.05$, compared with the other 2 groups. Data are shown as mean \pm SEM.

cast nephropathy, but did not undergo kidney biopsy. The $\kappa 6$ FLC was obtained from a patient who had Waldenström macroglobulinemia and progressive renal failure, but did not have a renal diagnosis from kidney biopsy. The monoclonal FLCs and the $\mathrm{V}_{\mathrm{L}}$ domain of the FLCs used in the overlay assays were provided by Alan Solomon (University of Tennessee Graduate School of Medicine, Knoxville, Tennessee, USA).

Yeast 2-bybrid assay. The yeast 2-hybrid system (MATCHMAKER GAL4 Two-Hybrid System 3; Clontech Laboratories) was used to detect binding interactions between THP and FLCs (16). A 72-bp fragment (encoding amino acid residues 218 to 241 of THP) and a 786 -bp fragment (encoding amino acid residues 148 to 410 of THP) were then cloned into the PGBKT7 expression vector for use in these studies. Both fragments contained the FLC-binding domain (amino acid residues 225 to 233, termed LCBD).

cDNA encoding $\kappa$ and $\lambda$ FLCs (37-39) were gifts from S. Louis Bridges Jr. (University of Alabama at Birmingham, Birmingham, Alabama, USA). The cDNA sequences were recorded in GenBank (LBPBLL2N, AF063768; ITPBLL56, AF063748; ITPBLL2, AF063741; LBPBLL2, AF063765; ITPBLL79, AF063755; ITPBLL86, AF063758; LKPBLL68, AF064501; ITPBLL11, AF063736; ITPBLL22, AF063742; LBPBLL2Q, AF063769; ITPBLL68, AF063750; ITPBLL69, AF063751; LKPBLL53, AF064498; LBPBLL7, AF063782; ITPBLL75, AF063753; LBPBLL2S, AF063770; ITPBL5, L40712; SSH23, M90809; ITPBL11, L40713; BCSyn9, L40686). The $V_{L}$ domains of 20 unique FLCs were individually cloned into PGADT7 for use in the yeast 2-hybrid experiments. Saccharomyces cerevisiae, AH109, was also cotransformed with plasmids that served as positive (pGBKT7-53/pGADT7-T) or negative (pGBKT7-Lam/pB42AD-T) experimental controls. pGBKT7-53 and PGADT7-T provided a positive control experiment by encoding proteins known to interact (murine p53 and SV40 large T antigen, respectively). pGBKT7-Lam encoded a fusion protein consisting of the DNA-binding domain and human lamin C, which does not interact with the SV40 large $\mathrm{T}$ antigen and thus served as a negative control. Double transformants

were selected in standard fashion using medium that lacked histidine and tryptophan. The transformants were incubated at $30^{\circ} \mathrm{C}$ for 4 days. Interactions between the 2 hybrid proteins were then tested by growth in galactose-containing, leucine-deficient medium ( -3 medium) and by $\beta$-gal plate and liquid culture assays. Reactions in the yeast 2-hybrid analyses were quantified by liquid culture assay of $\beta$-gal activity using $o$-nitrophenyl $\beta$-D-galactopyranoside (GE Healthcare Biosciences) as the substrate. This approach has been shown to correlate with interactions quantified using other biochemical methods (40).

Peptide competition experiments. To quantify the inhibitory capability of the competitor peptide of interest in preventing THP-FLC binding, a competitive ELISA was performed as described previously (16). In these experiments, the plates were initially coated with human FLCs, $0.2 \mu \mathrm{M}$, and blocked using 1\% BSA in PBS. Biotinylated human THP, $0.1 \mu \mathrm{M}$ in PBS, was then added, along with the competitor peptides in concentrations that ranged from 0 to $333 \mu \mathrm{M}$. After washing, the reaction was developed using avidin conjugated with HRP, 1:10000 dilution in PBS, followed by azino-bis(3-ethylbenzthiazoline-6-sulfonic acid). The optical density was determined at $405 \mathrm{~nm}$.

Protein expression and purification. The cDNA constructs for full-length human soluble THP and the $\mathrm{V}_{\mathrm{L}}$ domain of ITPBLL86 were each ligated into the pFN19K HaloTag T7 SP6 Flexi Vector (Promega Corp.), and the 2 fusion proteins (HaloTag attached to the THP and to the $V_{L}$ domain) were expressed using a cell-free system (TNT SP6 High-Yield Wheat Germ Protein Expression System; Promega Corp.). The proteins were purified by covalent immobilization using the attached HaloTag and HaloLink Resin (HaloTag Protein Purification System; Promega Corp.). A His-tagged TEV Protease was then used to cleave the HaloTag from the proteins; the Histag permitted subsequent removal of the protease using His-Link Resin. The estimated purity was greater than $95 \%$ by SDS-PAGE analysis.

Nonisotopic protein labeling. To biotin-label the $\mathrm{V}_{\mathrm{L}}$ fragment, the Transcend Non-Radioactive Translation Detection System (Promega Corp.) was employed. This system labeled the protein by permitting incorporation of biotin-labeled lysines during translation.

Overlay (far-Western) assays. Experiments were performed using a protocol previously reported (16). Following electrophoresis using a 10\% SDS-PAGE gel under nonreducing conditions, an FLC and the $\mathrm{V}_{\mathrm{L}}$ domain obtained from the same FLC were transferred to Immobilon PVDF. After washing and blocking, the membrane containing these proteins was incubated in PBS containing human THP, approximately $0.2 \mu \mathrm{mol} / \mathrm{l}$. After additional washes, the membrane was exposed to rabbit anti-human THP (Biomedical Technologies Inc.) and then HRP-conjugated anti-rabbit IgG, with development proceeding in standard fashion.

For the peptide competition experiments, equal and efficient transfer was confirmed by staining with MemCode Reversible Protein Stain (Ther-

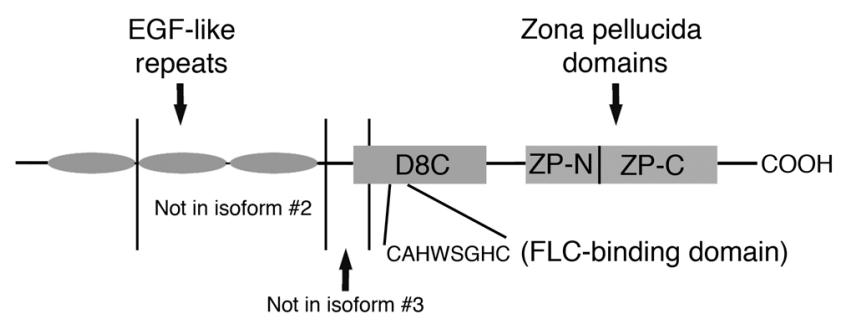

\section{Figure 8}

Schematic depicting the different domains on THP as well as potential alternative splice sites (represented by vertical lines). The FLC-binding domain (LCBD) lies between the EGF-like repeats and the zona pellucida domains. 
mo Fisher Scientific Pierce Protein Products). The PVDF membrane containing recombinant THP was then cut into strips, which were destained, washed, and blocked, then incubated with $\times 100, \times 30, \times 10, \times 3$, or $\times 1$ molar amounts (relative to bound THP) of the competitor cyclic peptide (AHXCLSADSSGSYLYVCKK). The strips were then incubated for 4 hours with the biotinylated $V_{L}$ domain of an FLC (ITPBLL86), followed by incubation for 2 hours with avidin-HRP. The membrane was then developed in standard fashion using Supersignal West Pico Chemiluminescent Substrate (Pierce Protein Products; Thermo Scientific).

In additional experiments, following electrophoresis of $\mathrm{\kappa} 2 \mathrm{FLC}$ in a $10 \%$ SDS-PAGE gel under reducing conditions, the FLC was transferred to PVDF membrane (Immobilon). Transfer was confirmed using MemCode Reversible Protein Stain. The PVDF membrane containing the $\kappa 2$ FLC was then cut into strips, which were destained, washed, and blocked, then probed with $0.2 \mu \mathrm{mol} / \mathrm{l}$ biotinylated human THP that had been preincubated with $\times 100, \times 30, \times 10, \times 3$, or $\times 1$ molar amounts (relative to THP) of the competitor cyclic peptide 1 (AHX-CLSADSSGSYLYVCKK) or control peptide (AHXCLSAHSSGSYLYVCKK) overnight at $4{ }^{\circ} \mathrm{C}$. After additional washes, the membranes were incubated for 2 hours with avidin-HRP. The membranes were then developed in standard fashion using Supersignal West Pico Chemiluminescent Substrate (Pierce Protein Products; Thermo Scientific) (16).

In vivo experiments. The Institutional Animal Care and Use Committee at the University of Alabama at Birmingham approved the project. Studies were conducted using male Sprague-Dawley (SD) rats (Harlan Laboratories) that were 4 to 6 weeks of age at the start of study. The rats were housed under standard conditions and fed a formulated $0.3 \% \mathrm{NaCl}$ diet (AIN-76A; Dyets Inc.).

Solomon et al. designed a rodent model that mimicked the human disease condition associated with monoclonal FLCs (41). The experimental protocol followed their approach, with the following modifications. To determine the in vivo efficacy of the cyclized peptide on prevention of AKI from monoclonal FLCs, on the morning of initiation of the study, rats were injected intraperitoneally with the experimental cyclized peptide 1 (AHX-CLSADSSGSYLYVCKK), $8 \mathrm{mg}(\sim 4.8 \mu \mathrm{mol})$ in PBS, or vehicle alone. Two hours later, each rat was injected intraperitoneally with monoclonal FLC ( $\kappa 3$ or $\lambda 2$ ), $20 \mathrm{mg}$ in PBS. Rats were placed individually into metabolic cages, and 24-hour urine samples were collected daily. On the second day of the study, the animals received a second intraperitoneal injection of the experimental peptide, $8 \mathrm{mg}$, or vehicle. On the final day of the study, the rats were anesthetized by intraperitoneal injection of pentobarbital sodium injection (Lundbeck Inc.), $50 \mathrm{mg} / \mathrm{kg} \mathrm{BW}$, and the kidneys were removed under aseptic conditions, fixed, and processed for frozen embedding as done previously (42). Light microscopy of H\&E staining for morphological analysis was performed using standard methods, and immunofluorescence microscopy was performed as described previously (43). In addition, blood was collected for determination of serum concentrations of urea nitrogen (BioAssay Systems) and creatinine, which was analyzed using an enzymatic assay (Genzyme Diagnostics).
To determine the in vivo efficacy of the cyclized peptide if administered after the monoclonal FLCs, serum was initially obtained for determination of creatinine concentration. Rats were then injected intraperitoneally with a monoclonal FLC ( $\kappa 2), 0.25 \mathrm{mg} / \mathrm{g}$ BW in PBS. Four hours later, rats were injected intraperitoneally with vehicle alone, experimental cyclized peptide 1 (AHX-CLSADSSGSYLYVCKK), or control peptide (AHX-CLSAHSSGSYLYVCKK), $0.1 \mathrm{mg} / \mathrm{g} \mathrm{BW}$ in PBS. On the following day of the study, serum was again obtained and the animals received a second intraperitoneal injection of the experimental or control peptide, $0.1 \mathrm{mg} / \mathrm{g} \mathrm{BW}$, or vehicle. On the final day of the study, the rats were anesthetized by intraperitoneal injection of pentobarbital sodium injection (Lundbeck Inc.), $50 \mathrm{mg} / \mathrm{kg}$ $\mathrm{BW}$, and the kidneys were removed under aseptic conditions, fixed, and processed for frozen embedding as described previously (42). Light and immunofluorescence microscopy were performed as described (43). Serum was collected for determination of creatinine concentration. In this study, serum creatinine was determined using liquid chromatography tandem mass spectrometry (Waters 2795 LC-MS/MS; Waters Corp.) (44).

To quantify cast formation, the number of casts present in 10 different $\times 300$ sections that contained medulla (defined as an absence of glomeruli in the section) was quantified in blinded fashion by one of the authors (L.M. Curtis).

Statistics. Data were expressed as mean \pm SEM. Significant differences between 2 groups were determined using an unpaired 2 -tailed $t$ test, and differences among multiple data sets were determined by ANOVA with standard post-hoc testing. Cast counts in the medulla for each experimental condition in vivo were analyzed by nonparametric ANOVA (KruskalWallis). SAS 9.2 was used for these statistical analyses (SAS Institute Inc.). $P<0.05$ was considered statistically significant.

Study approval. The Institutional Animal Care and Use Committee at the University of Alabama at Birmingham approved the animal protocol. The Birmingham VA Institutional Review Board approved the human protocol. Participants provided informed consent to collect their urine samples for protein purification.

\section{Acknowledgments}

NIH grants R01 DK046199 and P30 DK079337 (George M. O'Brien Kidney and Urological Research Centers Program) and the Office of Research and Development, Medical Research Service, Department of Veterans Affairs, Washington DC, USA, supported this research.

Received for publication January 20, 2011, and accepted in revised form February 1, 2012.

Address correspondence to: Paul W. Sanders, Division of Nephrology/Department of Medicine, 642 Lyons-Harrison Research Building, 1530 Third Avenue, South, University of Alabama at Birmingham, Birmingham, Alabama 35294-0007, USA. Phone: 205.934.3589; Fax: 205.975.6288; E-mail: psanders@uab.edu.
1. Sanders PW, Herrera GA, Galla JH. Human Bence Jones protein toxicity in rat proximal tubule epithelium in vivo. Kidney Int. 1987;32(6):851-861.

2. Sanders PW, Herrera GA, Chen A, Booker BB, Galla JH. Differential nephrotoxicity of low molecular weight proteins including Bence Jones proteins in the perfused rat nephron in vivo. J Clin Invest. 1988;82(6):2086-2096.

3. Sanders PW, Herrera GA, Kirk KA, Old CW, Galla $\mathrm{JH}$. Spectrum of glomerular and tubulointerstitial renal lesions associated with monotypical immunoglobulin light chain deposition. Lab Invest. 1991; 64(4):527-537.

4. Arimura A, Li M, Batuman V. Potential protective action of pituitary adenylate cyclase-activating polypeptide (PACAP38) on in vitro and in vivo models of myeloma kidney injury. Blood. 2006;107(2):661-668.

5. Pote A, Zwizinski C, Simon EE, Meleg-Smith S, Batuman V. Cytotoxicity of myeloma light chains in cultured human kidney proximal tubule cells. Am J Kidney Dis. 2000;36(4):735-744.

6. Sanders PW, Booker BB, Bishop JB, Cheung HC Mechanisms of intranephronal proteinaceous cast formation by low molecular weight proteins. J Clin Invest. 1990;85(2):570-576.

7. Sanders PW, Booker BB. Pathobiology of cast nephropathy from human Bence Jones proteins. J Clin Invest. 1992;89(2):630-639.
8. Weiss JH, et al. Pathophysiology of acute BenceJones protein nephrotoxicity in the rat. Kidney Int. 1981;20(2):198-210.

9. Greenlee RT, Murray T, Bolden S, Wingo PA. Cancer statistics, 2000. CA Cancer J Clin. 2000;50(1):7-33.

10 . Kyle RA, et al. Review of 1027 patients with newly diagnosed multiple myeloma. Mayo Clin Proc. 2003; 78(1):21-33.

11. Abbott KC, Agodoa LY. Multiple myeloma and light chain-associated nephropathy at end-stage renal disease in the United States: patient characteristics and survival. Clin Nephrol. 2001;56(3):207-210.

12. Blade J, et al. Renal failure in multiple myeloma: presenting features and predictors of outcome in 
94 patients from a single institution. Arch Intern Med. 1998;158(17):1889-1893.

13. Leung N, et al. Improvement of cast nephropathy with plasma exchange depends on the diagnosis and on reduction of serum free light chains. Kidney Int. 2008;73(11):1282-1288.

14. Huang Z-Q, Kirk KA, Connelly KG, Sanders PW. Bence Jones proteins bind to a common peptide segment of Tamm-Horsfall glycoprotein to promote heterotypic aggregation. J Clin Invest. 1993; 92(6):2975-2983.

15. Huang Z-Q, Sanders PW. Localization of a single binding site for immunoglobulin light chains on human Tamm-Horsfall glycoprotein. J Clin Invest. 1997;99(4):732-736

16. Ying W-Z, Sanders PW. Mapping the binding domain of immunoglobulin light chains for Tamm-Horsfall protein. Am J Pathol. 2001;158(5):1859-1866.

17. Fields S, Song O. A novel genetic system to detect protein-protein interactions. Nature. 1989. 340(6230):245-246.

18. Serafini-Cessi F, Malagolini N, Cavallone D. Tamm-Horsfall glycoprotein: biology and clinical relevance. Am J Kidney Dis. 2003;42(4):658-676.

19. Pennica D, et al. Identification of human uromodulin as the Tamm-Horsfall urinary glycoprotein. Science. 1987;236(4797):83-88.

20. Yang $\mathrm{H}$, Wu C, Zhao S, Guo J. Identification and characterization of D8C, a novel domain present in liver-specific LZP, uromodulin and glycoprotein 2 , mutated in familial juvenile hyperuricaemic nephropathy. FEBS Lett. 2004;578(3):236-238.

21. Schaeffer C, Santambrogio S, Perucca S, Casari G, Rampoldi L. Analysis of uromodulin polymerization provides new insights into the mechanisms regulating ZP domain-mediated protein assembly. Mol Biol Cell. 2009;20(2):589-599.

22. Jovine L, Qi H, Williams Z, Litscher E, Wassarman $\mathrm{PM}$. The ZP domain is a conserved module for polymerization of extracellular proteins. Nat Cell Biol. 2002;4(6):457-461.
23. Ota $\mathrm{T}$, et al. Complete sequencing and characterization of 21,243 full-length human cDNAs. Nat Genet. 2004;36(1):40-45.

24. Gerhard DS, et al. The status, quality, and expansion of the NIH full-length cDNA project: the Mammalian Gene Collection (MGC). Genome Res. 2004;14(10B):2121-2127.

25. Ceroni A, Passerini A, Vullo A, Frasconi P. DISULFIND: a disulfide bonding state and cysteine connectivity prediction server. Nucleic Acids Res. 2006; 34(Web Server issue):W177-W181.

26. Ferre F, Clote P. DiANNA: a web server for disulfide connectivity prediction. Nucleic Acids Res. 2005; 33(Web Server issue):W230-W232.

27. Gordon MS, Schmidt MW. Advances in electronic structure theory: GAMESS a decade later. In: Dykstra CE, Frenking G, Kim KS, Scuseria GE, eds. Theory And Applications Of Computational Chemistry: The First Forty Years. Amsterdam, The Netherlands: Elsevier; 2005:1167-1189.

28. Ren P, Ponder JW. Polarizable atomic multipole water model for molecular mechanics simulation. JPhys Chem B. 2003;107(24):5933-5947.

29. Ponder JW, et al. Current status of the AMOEBA polarizable force field. J Phys Chem B. 2010 114(8):2549-2564

30. Huang Z-Q, Sanders PW. Biochemical interaction of Tamm-Horsfall glycoprotein with Ig light chains. Lab Invest. 1995;73(6):810-817.

31. Holland MD, Galla JH, Sanders PW, Luke RG. Effect of urinary $\mathrm{pH}$ and diatrizoate on Bence Jones protein nephrotoxicity in the rat. Kidney Int. 1985; 27(1):46-50.

32. Knudsen LM, Hjorth M, Hippe E. Renal failure in multiple myeloma: reversibility and impact on the prognosis. Nordic Myeloma Study Group. Eur J Haematol. 2000;65(3):175-181.

33. Ludwig $H$, et al. Light chain-induced acute renal failure can be reversed by bortezomib-doxorubicindexamethasone in multiple myeloma: results of a phase II study. J Clin Oncol. 2010;28(30):4635-4641.
34. Basnayake K, et al. Resolution of cast nephropathy following free light chain removal by haemodialysis in a patient with multiple myeloma: a case report. J Med Case Reports. 2008;2:380.

35. Hutchison CA, et al. Treatment of acute renal failure secondary to multiple myeloma with chemotherapy and extended high cut-off hemodialysis. Clin J Am Soc Nephrol. 2009;4(4):745-754.

36. Ying W-Z, Sanders PW. Expression of Tamm-Horsfall glycoprotein is regulated by dietary salt in rats. Kidney Int. 1998;54(4):1150-1156.

37. Bridges SL Jr. Frequent $\mathrm{N}$ addition and clonal relatedness among immunoglobulin lambda light chains expressed in rheumatoid arthritis synovia and PBL, and the influence of V lambda gene segment utilization on CDR3 length. Mol Med. 1998;4(8):525-553.

38. Bridges SL Jr, et al. Somatic mutation and CDR3 lengths of immunoglobulin $\kappa$ light chains expressed in patients with rheumatoid arthritis and in normal individuals. J Clin Invest. 1995;96(2):831-841.

39. Stuber F, et al. A rheumatoid factor from a normal individual encoded by VH2 and V kappa II gene segments. Arthritis Rheum. 1992;35(8):900-904.

40. Estojak J, Brent R, Golemis EA. Correlation of twohybrid affinity data with in vitro measurements. Mol Cell Biol. 1995;15(10):5820-5829.

41. Solomon A, Weiss DT, Kattine AA. Nephrotoxic potential of Bence Jones proteins. N Engl J Med. 1991;324(26):1845-1851.

42. Curtis LM, Chen S, Chen B, Agarwal A, Klug CA, Sanders PW. Contribution of intrarenal cells to cellular repair after acute kidney injury: subcapsular implantation technique. Am J Physiol Renal Physiol. 2008;295(1):F310-F314

43. Novak L, Cook WJ, Herrera GA, Sanders PW. ALamyloidosis is underdiagnosed in renal biopsies. Nephrol Dial Transplant. 2004;19(12):3050-3053.

44. Takahashi N, Boysen G, Li F, Li Y, Swenberg JA. Tandem mass spectrometry measurements of creatinine in mouse plasma and urine for determining glomerular filtration rate. Kidney Int. 2007;71(3):266-271. 